\title{
ON THE INSET OF A CONVERGENCE DOMAIN
}

\author{
SHEN-YUE KUAN
}

\begin{abstract}
Let $\boldsymbol{A}$ be a conservative matrix. The main purpose of this article is to give a negative answer to the open question $E$ raised by Macphail and Wilansky [1], namely, "If $\Lambda_{A}^{\perp}=I_{A}$, must $\Lambda_{D}^{\perp}=I_{D}$ for every matrix $D$ with $c_{D}=c_{A}$ ?".
\end{abstract}

The main purpose of this short article is to give a negative answer to the open question $E$ which was raised by M. S. Macphail and A. Wilansky in [1].

As usual, we use $c, c_{0}, l$, respectively, for the set of all convergent sequences, null sequences, absolutely summable sequences. Let $A$ be an infinite matrix. The convergence domain $c_{A}=\{x: A x \in c\}$ is an $F K$ space. We assume $A$ conservative, that is, $c \subset c_{A}$. With $a_{k}$ denoting the $k$ th column limit of $A$, we define the inset

$$
I_{A}=\left\{x \in c_{A}: \sum_{k} a_{k} x_{k} \text { converges }\right\}
$$

and

$$
\Lambda_{A}^{\perp}=\left\{x \in I_{A}: \lim _{A} x=\sum a_{k} x_{k}\right\} .
$$

Each $f \in c_{A}^{\prime}$ has a representation

$$
f(x)=\mu \lim _{A} x+t(A x)+s x \quad\left(t \in l, s \in c_{A}^{\beta}\right) .
$$

Let $A$ be the matrix defined by

$$
(A x)_{2 n}=0, \quad(A x)_{2 n-1}=-\frac{2}{n} x_{n}+\frac{1}{n} x_{n+1} .
$$

Plainly $\lim _{A} x=0\left(x \in c_{A}\right)$ so $\mu$ is not unique, $I_{A}=c_{A}, \Lambda_{A}^{\perp}=I_{A}$. Define $g(x)=\lim _{n} 2^{-n} x_{n}$ on $c_{A}$. Let $y=\left(y_{n}\right)=A x$. It is easy to see that

$$
\sum_{k=1}^{n} 2^{-(k+1)} k y_{2 k-1}=-2^{-1} x_{1}+2^{-(n+1)} x_{n+1}
$$

Put $t=\left(t_{n}\right)$ where $t_{2 k}=0, t_{2 k-1}=2^{-(k+1)} k, k=1,2,3, \ldots$ Then $t \in l$. From (2) the function $g$ can be expressed as

$$
g(x)=t(A x)+2^{-1} x_{1}
$$

Received by the editors January 4, 1978.

AMS (MOS) subject classifications (1970). Primary 40H05, 46A45.

Key words and phrases. Convergence domain, conservative matrix, the inset.

๑) American Mathematical Society 1978 
Hence $g \in c_{A}^{\prime}$ and so its kernel $g^{\perp}$ is closed and $g^{\perp} \supset \bar{c}_{0}$. On the other hand the sequence $u=\left(2^{n}\right)$ belongs to $c_{A}$ and $g(u)=1$, so $u \in c_{A} \backslash \bar{c}_{0}$. There exists $f \in c_{A}^{\prime}$ with $f(u) \neq 0$ and $f=0$ on $\bar{c}_{0}$. By $[2$, Satz 5.3] there exists a matrix $D$ with $c_{D}=c_{A}$ and $f=\lim _{D}$ since $\mu$ is not unique for $A$. Now $d_{k}=\lim _{D} e^{k}=$ $f\left(e^{k}\right)$, where $e^{k}$ denotes the sequence $(0, \ldots, 0,1,0, \ldots)$ with the 'one' in the $k$ th position. Hence $I_{D}=c_{D}=c_{A}$. But

$$
\Lambda_{D}(u)=\lim _{D} u-\sum d_{k} u_{k}=\lim _{D} u=f(u) \neq 0 .
$$

Thus $\Lambda_{D}^{\frac{1}{D}} \neq I_{D}$.

\section{REFERENCES}

1. M. S. Macphail and A. Wilansky, Linear functionals and summability invariants, Canad. Math. Bull. 17 (1974), 233-242.

2. K. Zeller, Allgemeine Eigenschaften von Limitierungsverfahren, Math. Z. 53 (1951), 463-487.

Department of Mathematics, National Central University, Chung Li, Tajwan, RepubLIC OF ChInN 